\title{
"You Can’t Be Outside Without Being Part of The Culture": Recommendations For Using Outdoor Recreation To Support International Student Transitions
}

\author{
Michelle K Brunette $^{\mathrm{a}, *}$, Callie Mady ${ }^{b}$, Tara-Lynn Scheffel ${ }^{b}$, Denyse Lafrance Horning ${ }^{b}$, and Maria \\ Cantalini-Williams ${ }^{b}$ \\ ${ }^{a}$ Laurentian University, Canada; ${ }^{b}$ Nipissing University, Canada \\ *Corresponding author: Email: mkbrunette@laurentian.ca \\ Address: Laurentian University, 935 Ramsey Lake Rd. Sudbury, ON P3E 2C6, Canada
}

Received 1/13/19; revised 3/14/19; revised 5/31/19; accepted 8/21/19

\section{Introduction}

Outdoor education and sport sociology researchers have associated participation in outdoor recreation with physical and mental health wellbeing, reduced anxiety, increased social participation, and increased cultural exchange (Stodolska 2015; Weng and Chiang 2014). International students appreciate opportunities to experience Canada's natural environment through activities, open spaces, and outdoor opportunities (Brunette, Lariviere, Schinke, Xing and Pickard 2011). The outdoor environment can benefit both the recruitment and wellbeing of students, yet international student expectations and experiences of Canadian outdoor recreation remain largely unexplored in research. An understanding of international student expectations and experiences is important, as unmet expectations and negative initial experiences can lead to poor student integration and attrition (Tinto 1999).

In this paper, the researchers draw on interdisciplinary fields to investigate the unique benefits of outdoor recreation in facilitating international student transitions to new study environments. Drawing on their findings, the researchers offer recommendations to universities to promote, adapt, and enhance the use of outdoor recreation to support international students on their campuses.

\section{Context}

\section{International Students in Canada}

International student enrolment in Canada increased 154\% from 2010 to 2018 (Canadian Bureau for International Education [CBIE] 2019). In 2018, Canada attracted 572,415 international students with the largest numbers of students arriving from India (30\%) and China (25\%) (CBIE 2019). Canadian universities find advantages in using the natural landscape in the recruitment of international students who appreciate Canada's abundance of outdoor spaces and activities, (Brunette, Lariviere, Schinke, Xing and Pickard 2011). A review of Canadian university websites revealed heavy use of images of the outdoor environment, including Brock's (2016) "vibrant region with beautiful natural surroundings” (para. 2) and Carleton's (2016) "park-like greenery, and extensive walking and biking trails" (para. 3).

\section{Cultural Transitions and Outdoor Recreation}

International students face challenges in their studies including, a) linguistic and language proficiency barriers (Kuo and Roysircar 2004; Zhang and Zhou 2010); b) social and sense of belonging difficulties (Houshmand, Spanierman and Tafarodi 2014; Shifman, Moss, D'Andrade, Zhou and Zhang 2014); and c) financial and access to employment barriers (Calder et al 2016; Scott, Safdar, Trilokekar and El Masri 2015). There are numerous challenges in making cross-cultural transitions, however, there are several established benefits of outdoor recreation that can help ease some of the transition barriers.

Cross-cultural transitions can be associated with physical and mental stress (Oberg 1954; Berry, Kim, Minde and Mok 1987), while participation in outdoor recreation is associated with improved physical and mental health wellbeing (Duvall 2011; Weng and Chiang 2014). Cultural transitions have been associated with depression and anxiety (Poyrazli and Grahame 2007), yet 
outdoor recreation contributes to improved mental health, reduced anxiety, and improved moods (Roe and Aspinall 2011). Adapting to new cultures lends to difficulties in navigating social relationships and a lack of a sense of belonging (Walseth 2006), while outdoor recreation offers opportunities to increase social participation, selfesteem, and cultural learning in a new community (Stodolska 2015). Outdoor recreation often increases physical activity, which can contribute to international students' ability to reduce stress, make social connections, improve language skills, and learn about the Canadian environment (Brunette, Lariviere, Schinke, Xing and Pickard 2011). Even just being near nature contributes to higher overall life and employment satisfaction (e.g., Tennessen and Cimprich 1995).

Some institutions use the Canadian natural environment to help welcome and support international students in their transition to Canada. For example, Trent University's 4-day camp experience includes canoeing and hiking as part of a mandatory orientation, and Laurentian University invites students to be part of summer and winter camping events. It is reasonable to consider the potential of the outdoor environment in supporting international students' transitions to new cultural environments.

\section{Methods}

Drawing on interdisciplinary fields including international education and sport sociology, international student expectations of the Canadian outdoors were compared to their lived experiences in their first year at a university in Northern Ontario, Canada.

\section{Theory to Inform Research Context and Design}

The exploration of student expectations was informed with Tinto's (1999) model of student integration which considers expectations as part of the holistic student experience and Oliver's (1980) expectancy-disconfirmation paradigm which assigns positive or negative values to met and unmet expectations. Understanding cross-cultural lived experiences was informed by Dewey's (1938) theories of experience, particularly in the intensity and direction (i.e., positive/negative) of emotional stirring that impacts value of experience, and Vygotsky's (1986) sociocultural theory in aiming to understand how experiences are shaped by social interactions in nonformal environments. Finally, the methods are also underpinned by theoretical foundations including van Manen's (1990) interpretive phenomenology which recognizes that participant and researcher interpretations of experiences, together, shape the study, and Hofstede's (2011) cultural value orientations in assessing how culture impacts expectations, experiences, and the researcher-participant interactions.

\section{Sequential Qualitative Dominant Mixed Methodology}

A sequential qualitative dominant mixed methodology was used, informed by Johnson, Onwuegbuzie and Turner (2007), starting with a survey for breadth of data and to identify emerging themes, and followed by a three-stage guided walk interview to gain greater depth in clarifying, revisiting, and reflecting on the data. The surveys used non-probability (non-random) sampling, with all new international students who participated in orientation invited to complete the survey during orientation activities throughout the first two weeks of the Fall semester.

Forty-seven (76\%) international students completed the survey to illuminate international student expectations of outdoor recreation. The final survey question asked students if they were interested in being part of follow up interviews that took place outdoors. All participants who indicated an interest in follow up interviews were contacted leading to seven international students participating in the three-stage interviews. Six participants were undergraduates and one was a graduate student, and the participants had six different first languages of communication (Arabic, Chinese, English, French, Malagasy, and Thai).

\section{Guided Walks}

A guided walk is a mobile interview method, which can ease participants into interviews by building rapport and situating the importance of place in the conversation (Evans and Jones, 2011). Guided walks are useful in studies with participants from diverse cultures, as they encourage open and pressure-free dialogue, and a balance of power between participants and researcher (Jones, Bunce, Evans, Gibbs and Hein, 2008). From a 
cross-cultural perspective, walking side-by-side helps reduce cultural misinterpretations of different uses of eye contact or physical distance (Kinlock and Metge, 2014). In the guided walks, I also adhered to guidelines for cross-cultural interviewing, including offering more interview time and allowing translation devices if needed (Cortazzi, Pilcher and Jin, 2011).

There were three stages of the guided walk interviews (approximately 1 hour per interview) between September and January. The timeline was set during participants' first year at the university. This early timing is important, as numerous researchers have linked unmet expectations and poor initial student experiences to student attrition (Kuh 2005). Prior to each guided walk, students received an email with the semi-structured interview questions for their review. In the postinterview phase, the participants received transcripts and initial themes for clarification and co-construction of meaning.

\section{Analysis and Findings}

Stage 1 was an analysis of the quantitative data collected through the international student survey, which informed follow up interviews. Stage 2 was an on-going thematic analysis of the interview data collected in the three-stage guided walks. The analysis of the first interview helped clarify perspectives shared through the survey, especially those related to student expectations. The second and third interview analyses focused on revisiting and reflecting on expectations relative to student experiences.

Oliver's (1980) expectancy-disconfirmation paradigm (EDP), a marketing theory, was used to classify participants' experiences as negative or positive based on how well expectations match experience. In Oliver's framework, positive experiences are those that confirm positive expectations or disconfirm negative expectations. According to Appleton-Knapp and Krentler (2006), EDP is "an appropriate means by which to investigate the relationship between student expectations and student satisfaction" (p. 256).

The initial survey provided insight into international student expectations of the Canadian outdoor environment. Most respondents (55\%) said the images profiled on university marketing materials encouraged them to study in Canada. Most (69\%) agreed that opportunities to participate in outdoor recreation impacted their decision to choose Canada as a destination of study, even though many (55\%) expected their participation in outdoor recreation to be different than in their home countries due to different weather and types of sport. $61 \%$ were very interested in participating in Canadian outdoor activities in the summer (e.g., canoeing, kayaking, swimming, hiking) and winter (e.g., skating, hockey, skiing).

Thematic analysis of the interview phases revealed both negative and positive experiences in the outdoors. Negative experiences related to a) Canada's cold weather, b) safety, and c) finding partners. $\mathrm{Li}$ described a challenge of Canadian winter, "I thought it would be very good with a lot of snow. It's complicated. I found myself wearing wrong clothes." Safety concerns arose with lack of familiarity with some activities. Mo provided this example, "I can't understand the baseball game so I just used my hand to catch a ball...I almost break my hand." One participant summarized the difficulty in finding partners: "it is hard to find people to go with." Some of the burden was driven by challenges in communicating with domestic students, as Fannie described; "I was thinking about going hiking with Canadian students because they are familiar with this area...but it's really hard to socialize with them."

Participants found it more difficult than they anticipated to communicate with Canadian students, but felt more at ease with other international students. Fannie explained: "sometimes international students seem more relaxed with other international students...I feel we are the same. I sometimes feel it is hard to talk to Canadian people." Participants expected that outdoor activity would provide an opportunity to communicate with domestic students, which, in reality, the participants' found to be a significant barrier to their participation.

Despite some of the challenges, outdoor recreation provided many positive experiences, including a) ease of access, b) opportunities to practice English, c) belonging in the Canadian community; and iv) an overall sense of wellbeing. Participants expected that finding outdoor 
equipment and access to recreation opportunities might have posed barriers to their participation, but instead they had positive experiences with easy access to equipment and finding free space to access the outdoors.

Karim shared his experience in finding outdoor opportunities, "it's not a challenge if you want to do it. There is a lot for you... convenient and it is all free." Outdoor recreation also provided participants a space to practice English in an informal environment. Fannie shared, "I am just trying to engage in the city, talk with people, and improve my English." Bohai described feeling part of the community after he participated in his first hockey game; "I went to play hockey... going to these activities is not just for the activities, but just to be with people." In the final of the three guided-walk, Ada reflected on the importance of being outdoors in Canada: "you can't be outside without being part of the culture."

Fannie described how the outdoors contributed to her overall sense of wellbeing, "I really like the views in Canada. When I go outside I feel nice... the feeling makes me feel better." Another participant, Bohai, shared that being outside boosted his mood; "inside is lonely. You feel helpless... Reading Week and Thanksgiving is really lonely, but when you walk outside in this country I feel more hopeful, I feel more comfortable, buoyant, elevated." Participating in outdoor recreation was a way for participants to manage the stressful transition of studying in a new country.

\section{Discussion and Recommendations to Universities}

Aligning with Tinto's (1999) model of student integration, student expectations shape their actions, interactions and experiences, and ultimately their decision to stay. It is important for universities to set the right expectations about the outdoor environment to support international students in their transitions. In planning outdoor recreation, international students should be consulted to consider cultural diversity and avoid activities that may be uncomfortable for some students (Purdie and Neill 1999). To ease participation, it is important to provide sufficient information for international students about the types of activities available, gear required, where to access activities, and safety (Brunette, Lariviere, Schinke, Xing and Pickard 2011).

Ways to support student recruitment and services arise from the stories shared by participants. Recommendations are linked to six phases of the international student experience; (a) recruitment, (b) preparation, (c) orientation, (d) planning, (e) delivery, and (f) evaluation.

\section{Recruitment}

1. Appeal to what international students need or want from their experiences at the destination university by highlighting desirable outdoor recreation opportunities in university marketing materials.

2. Clearly define expectations of the outdoors, including climate, clothing and gear, and opportunities to participate.

3. Define potential types of social interactions between international and domestic students.

\section{Preparation}

1. Share links to Google Earth for students to virtually experience the campus and surroundings.

2. Invite the local community to participate in a donation drive for climate appropriate clothing and recreation gear for international students.

3. Develop intercultural training for outdoor education leaders to increase cultural selfawareness and cross-cultural competency to respond to the needs of culturally diverse participants.

\section{Planning}

1. Plan group outdoor recreation activities in all seasons, adhering to appropriate risk management protocols in coordination with the university's safety office.

2. Invite upper year international students to participate in the planning of outdoor activities to identify barriers to participation.

3. Share a schedule of accessible and appropriate outdoor recreation opportunities to help students located convenient activities and partners.

\section{Orientation}


1. Clarify student expectations of outdoor recreation opportunities to tailor future programming appropriately.

2. Include outdoor recreation in the orientation schedule.

3. Introduce students to outdoor recreation services/opportunities, with an emphasis on free campus/community activities.

4. Remind students of climate-appropriate clothing or gear and where to locate these items.

\section{Delivery}

1. Offer training/instruction in using equipment, and adhere to risk management protocols.

2. Bring extra outdoor gear for students who may not have planned appropriately for the weather conditions.

3. Check on students' comfort level during the activity including asking about their feeling of safety, warmth, and interest in continuing the activity.

\section{Evaluation}

1. Collect feedback from students and leaders to evaluate satisfaction with activities and social interactions, and to inform programming revisions.

2. Requesting permission to share comments in future marketing materials.

These recommendations aim to guide universities in recognizing and stimulating positive international student experiences in the outdoors, and to contribute to an overall sense of belonging and wellbeing.

\section{Implications and Future Exploration}

The recommendations arise from a study in a Canadian university context with one cohort of international students in Northern Ontario. Despite the limited focus, the researchers encourage post-secondary institutions in all regions to understand and clarify international student expectations and consider the outdoor environment as part of international student retention efforts. This is not to imply that all student expectations should be positive, nor, that all lived experiences will be positive. More importantly, to best support international students, expectations need to be clear and realistic. Different universities may need to manage expectations differently and offer unique supports to international students to help them experience the benefits of the natural environment.

The outdoor environment can act as unique, and important, space to help facilitate international student transitions to new study environments. The recommendations are aimed at enhancing the use outdoor recreation as part of orientation programs, and ultimately to support international students. 


\section{References}

Appleton-Knapp, Sara, and Kathleen Krentler. "Measuring student expectations and their effects on satisfaction: The importance of managing student expectations." Journal of Marketing Education 28, no. 3 (2006): 254-264.

Berry, John, Uichol Kim, Thomas Minde, and Doris Mok. "Comparative studies of acculturative stress." International Migration Review 21, no. 3 (1987): 491-511.

Brock. (2016, September 22). Welcome to Brock. Retrieved from http://brocku.ca/flipbook/brockbook-int-en/

Brunette, Michelle K., Michel Lariviere, Robert J. Schinke, Xiaoyan Xing, and Pat Pickard. "Fit to belong: Activity and acculturation of Chinese students." Journal of Sport Behavior 34, no. 3 (2011).

Calder, Moira, Solina Richter, Yuping Mao, Katharina Burns, Ramadimetja Mogale, and Margaret Danko. "International students attending Canadian universities: Their experiences with housing, finances, and other issues." Canadian Journal of Higher Education 46, no. 2 (2016): 92-110.

Canadian Bureau for International Education (CBIE). (2019). Facts and figures. Canadian Bureau for International Education. Retrieved May 3, 2019, from https://cbie.ca/infographic/

Carleton. (2016, September 22). Life in Ottawa: a great place to live and learn. Retrieved from http://admissions.carleton.ca/student-

life/lifeinottawa/

Cortazzi, Martin, Nick Pilcher, and Lixian Jin. "Language choices and 'blind shadows': investigating interviews with Chinese participants." Qualitative Research 11, no. 5 (2011): 505-535.

Dewey, John. "Experiential education." New York: Collier (1938).

Duvall, Jason. "Enhancing the benefits of outdoor walking with cognitive engagement strategies." Journal of Environmental Psychology 31, no. 1 (2011): 27-35.
Hofstede, Geert. "Dimensionalizing cultures: The Hofstede model in context." Online Readings in Psychology and Culture 2, no. 1 (2011): 8.

Houshmand, Sara, Lisa Spanierman, and Romin Tafarodi. "Excluded and avoided: Racial microaggressions targeting Asian international students in Canada." Cultural Diversity and Ethnic Minority Psychology 20, no. 3 (2014): 377.

Johnson, R. Burke, Anthony J. Onwuegbuzie, and Lisa A. Turner. "Toward a definition of mixed methods research." Journal of Mixed Methods Research 1, no. 2 (2007): 112-133.

Jones, Phil, Griff Bunce, James Evans, Hannah Gibbs, and Jane Ricketts Hein. "Exploring space and place with walking interviews." Journal of Research Practice 4, no. 2 (2008): D2.

Kinloch, Patricia, and Joan Metge. Talking past each other: problems of cross cultural communication. Victoria University Press, 2014.

Kuh, George D. "Student engagement in the first year of college." Challenging and supporting the first-year student: A handbook for improving the first year of college (2005): 86-107.

Kuo, Ben $\mathrm{CH}$, and Gargi Roysircar. "Predictors of acculturation for Chinese adolescents in Canada: Age of arrival, length of stay, social class, and English reading ability." Journal of Multicultural Counseling and Development 32, no. 3 (2004): 143 154.

Oberg, Kalervo. "Cultural shock: Adjustment to new cultural environments." Practical Anthropology 4 (1960): 177-182.

Oliver, Richard L. "A cognitive model of the antecedents and consequences of satisfaction decisions." Journal of Marketing Research 17, no. 4 (1980): 460-469.

Poyrazli, Senel, and Kamini M. Grahame. "Barriers to adjustment: Needs of international students within a semi-urban campus community." Journal of Instructional Psychology 34, no. 1 (2007): 28.

Purdie, Nola, and James Neill. "Japanese students downunder: Is Australian outdoor education relevant to other cultures?" Journal of Outdoor and Environmental Education 4, no. 1 (1998): 48-57. 
Roe, Jenny, and Peter Aspinall. "The restorative benefits of walking in urban and rural settings in adults with good and poor mental health." Health \& Place 17, no. 1 (2011): 103-113.

Scott, Colin, Saba Safdar, Roopa Desai Trilokekar, and Amira El Masri. "International Students as' Ideal Immigrants' in Canada: A disconnect between policy makers' assumptions and the voices of international students" Comparative and International Education 43, no. 3 (2015): 1.

Shifman, Robbie, Katelyn Moss, Giselle D’Andrade, Jessica Eichel, and Scott Forrester. "A comparison of constraints to participation in intramural sports between international and noninternational students." Recreational Sports Journal 36, no. 1 (2012): 2-12.

Stodolska, Monika. "Recreation for all: Providing leisure and recreation services in multi-ethnic communities." World Leisure Journal57, no. 2 (2015): 89-103.

Tennessen, Carolyn M., and Bernadine Cimprich. "Views to nature: Effects on attention." Journal of Environmental Psychology 15, no. 1 (1995): 77-85.

Tinto, Vincent. "Taking retention seriously: Rethinking the first year of college." NACADA Journal 19, no. 2 (1999): 5-9.

van Manen, Max. Researching lived experience: Human science for an action sensitive pedagogy. Routledge, 2016.

Vygotsky, Lev S. "Thought and language (A. Kozulin, trans.)." (1986).

Walseth, Kristin. "Sport and belonging." International Review for the Sociology of Sport 41, no. 3-4 (2006): 447-464.

Weng, Pei-Yi, and Yen-Cheng Chiang. "Psychological restoration through indoor and outdoor leisure activities." Journal of Leisure Research 46, no. 2 (2014): 203-217.

Zhou, George, and Zuochen Zhang. "A study of the first year international students at a Canadian university: Challenges and experiences with social integration." Comparative and International Education 43, no. 2 (2014): 7.
Zhang, Zuochen, and George Zhou. "Understanding Chinese international students at a Canadian university: Perspectives, expectations, and experiences." Comparative and International Education 39, no. 3 (2010): 43-58. 